\title{
MUTUAL RECIPROCAL INTERCONNECTION OF RELATIONS DESCRIBING CREEP, VIELD STRESS AND STRESS RELAXATION
}

The steady-state creep rate increases with testing temperature according to the Arrhenius law and its increase with applied stress is usually described by the power law. Simple multiplication of these two laws leads to the dependence of steady-state creep rate on both the variables. Apparent activation energy and stress sensitivity parameter perform in the combined law as constants but most measurements show their dependence on some of the variables. An equation respecting these experimental facts and simultaneously also based on the Arrhenius equation and the power law is derived and verified using some published results of creep tests. It can be presented geometrically by hyperbolic paraboloid. Resulting from this equation, the dependence of yield stress on temperature and strain rate and also an equation describing the relaxation curves are deduced.

\section{Introduction}

The steady-state creep rate $\dot{\varepsilon}$ at a given applied stress $\sigma$ increases with temperature $T$ according to the Arrhenius equation (e.g. Cadek [1])

$$
\dot{\varepsilon} \propto \exp \left(-\frac{Q_{a}}{R T}\right) \text { where } Q_{a}=\left.\frac{\partial \ln \dot{\varepsilon}}{\partial(-1 / R T)}\right|_{\sigma=\text { const. }}
$$

is the apparent activation energy of creep and $R$ is the universal gas constant. The increase of the creep rate at a given temperature with applied stress is mostly described by the power law

$$
\dot{\varepsilon} \propto \sigma^{\prime \prime} \text { where } n=\left.\frac{\partial \ln \dot{\varepsilon}}{\partial \ln \sigma}\right|_{T=\text { const. }}
$$

is the stress sensitivity parameter (see Garofalo [2]). Combining Eqs (1) and (2), an equation

$$
\dot{\varepsilon}(\sigma, T)=C\left(\frac{\sigma}{G}\right)^{\prime \prime} \exp \left(-\frac{Q_{a}}{R T}\right)
$$

is obtained describing the dependence of creep rate on both the variables determining experimental conditions (e.g. Cadek [1], Sherby [3]). The main role of elasticity modulus in shear $G$ is to avoid the problems with physical units in the case when $n$ is not equal to integer.

The values of apparent activation energy and of stress sensitivity parameter are implicitly assumed to be constant but nearly all the results of creep tests show that the apparent activation energy depends on applied stress and the stress sensitivity parameter depends on temperature. The solution of these contradictions is the main aim of the present paper.
During several last decades the main attention was paid to physical approach: various creep mechanisms were described by means of many different models. Each of the models leads to certain integer value of stress sensitivity parameter in the range from 1 to 7 (see e.g. Cadek [1]). The results of creep experiments lead to real values of this parameter sometimes substantially overreaching the value of 10 . The approach presented in this paper is fully phenomenological but without any limitation of the stress sensitivity parameter value.

\section{New relation for steady-state creep rate}

Apparent activation energy $Q_{a}$ is defined for constant applied stress and stress sensitivity parameter $n$ is defined for constant temperature, see Eqs (1) and (2). Therefore, dependences $Q_{a}=Q_{a}(\sigma)$ and $n=n(T)$ can be considered. Considering Eqs (1) and (2), the creep rate can be concurrently expressed in the following two ways

$$
\dot{\varepsilon}(\sigma, T)=A(\sigma) \exp \left[-\frac{Q_{a}(\sigma)}{R T}\right] \text { and } \dot{\varepsilon}(\sigma, T)=B(T) \sigma^{n(T)}
$$

because the factor of proportionality $A$ can be dependent (at least principally) on applied stress and the factor of proportionality $B$ can be dependent on temperature. But both relations (4) should be equivalent in principle. To derive the common equation, the values $\sigma=1(\mathrm{MPa})$ and/or $T \rightarrow \infty$ are introducing, for which Eqs (4) turn substantially simpler [4]

$$
\begin{aligned}
& \dot{\varepsilon}(1, T)=A(1) \exp \left[-\frac{Q_{a}(1)}{R T}\right]=B(T) \\
& \dot{\varepsilon}(\sigma, \infty)=A(\sigma)=B(\infty) \sigma^{n(\infty)}
\end{aligned}
$$

\footnotetext{
* Jan Kohout ${ }^{1}$, Vojtech Hruby ${ }^{2}$

${ }^{1}$ Department of Mathematics and Physics, Faculty of Military Technology, University of Defence, Brno, Czech Republic, E-mail: jan.kohout@unob.cz

${ }^{2}$ Department of Mechanical Engineering, Faculty of Military Technology, University of Defence, Brno, Czech Republic
} 


$$
\dot{\varepsilon}(1, \infty)=A(1)=B(\infty)
$$

Using new declarations (and taking into account negative values of $p$ )

$$
\begin{aligned}
& Q_{a}(1)=E \ln \sigma_{0}, n(\infty)=p=-\frac{E}{R T_{0}}, \\
& \text { and } \frac{B(\infty)}{\sigma^{n(\infty)}}=\dot{\varepsilon}_{0}
\end{aligned}
$$

the apparent activation energy as well as the stress sensitivity parameter can be expressed as

$$
Q_{a}(\sigma)=E \ln \frac{\sigma_{0}}{\sigma}, \text { and } n(T)=\frac{E}{R}\left(\frac{1}{T}-\frac{1}{T_{0}}\right)
$$

and the form of the searched dependence is

$$
\dot{\varepsilon}(\sigma, T)=\dot{\varepsilon}_{0}\left(\frac{\sigma_{0}}{\sigma}\right)^{E / R T_{0}} \exp \left(-\frac{E}{R T} \ln \frac{\sigma_{0}}{\sigma}\right) \equiv \dot{\varepsilon}_{0}\left(\frac{\sigma_{0}}{\sigma}\right)^{\frac{E}{R}\left(\frac{1}{T}-\frac{1}{T_{0}}\right)}
$$

Eq. (7) explains the meaning of parameter $E$ : it represents apparent activation energy for applied stress $\sigma=\sigma_{0} / \mathrm{e}(\mathrm{e}=$ $=2.7182818 \ldots$ is the base of natural logarithms).

\section{Geometrical explication of final equation and its parameters}

Logarithmic form of Eq. (8)

$$
\ln \dot{\varepsilon}(\sigma, T)=\frac{E}{R}\left(\frac{1}{T}-\frac{1}{T_{0}}\right) \cdot\left(\ln \sigma-\ln \sigma_{0}\right)+\ln \dot{\varepsilon}_{0}
$$

obtains in coordinates

$$
\begin{aligned}
& x=\frac{1}{T}, x_{0}=\frac{1}{T_{0}}, y=\ln \sigma, y_{0}=\ln \sigma_{0}, \\
& z=\ln \dot{\varepsilon}, \text { and } z_{0}=\ln \dot{\varepsilon}_{0}
\end{aligned}
$$

a simple form

$$
z(x, y)=a\left(x-x_{0}\right)\left(y-y_{0}\right)+z_{0}, \text { where } a=E / R
$$

which represents the equation of hyperbolic paraboloid with the origin in point $\left(x_{0}, y_{0}, z_{0}\right)$. This fact explains the meaning of parameters $T_{0}, \sigma_{0}$ and $\dot{\varepsilon}_{0}$. The equation of hyperbolic paraboloid (11) written for $(0,0,0)$ origin

$$
z(x, y)=a x y
$$

differs from the most usual form

$$
x^{2}-y^{2}=b z, \text { where } b=2 / a
$$

because the paraboloids (12) and (13) are turned one to the other by $45^{\circ}$ round the z-axis.

Analyzing Eq. (8) it can be said:

1. For the temperature $T=T_{0}$ the creep rate is independent of applied stress (arbitrary number raised to 0 is equal to 1 ).
Therefore the curves $\dot{\varepsilon}=f(T)$ for various applied stresses intersect in one point and its coordinates are $T_{0}, \dot{\varepsilon}_{0}$. In the $1 / T-\ln \dot{\varepsilon}$ fit these curves are represented by straight lines with the slope equal to the ratio $Q_{a} / R$, see Eq. (1).

2. For the applied stress $\sigma=\sigma_{0}$ the creep rate is independent of temperature (1 raised to arbitrary number is equal to 1 ). Therefore the curves $\dot{\varepsilon}=f(\sigma)$ for various temperatures intersect in one point with coordinates $\sigma_{0}, \dot{\varepsilon}_{0}$. In the $\ln \sigma-\ln \dot{\varepsilon}$ fit these curves are represented by straight lines with slope equal to stress sensitivity parameter $n$, see Eq. (2).

Representation of the curves $\dot{\varepsilon}=f(T)$ and $\dot{\varepsilon}=f(\sigma)$ by straight lines in the fits $1 / T-\ln \dot{\varepsilon}$ and $\ln \sigma-\ln \dot{\varepsilon}$ follows directly from the fact that Eq. (8) describes hyperbolic paraboloid in coordinates (10). Depicting of Eq. (8) not only in 3D by hyperbolic paraboloid but also in 2D by families of straight lines if coordinates (10) are used, defines unambiguously the geometrical meaning of parameters $T_{0}, \sigma_{0}$ and $\dot{\varepsilon}_{0}$ as well as the meaning of the fourth parameter $E=Q_{a}\left(\sigma_{0} / \mathrm{e}\right)$ describing in fact the scale factor of the third axis with respect to the first two axes. It is the greatest advantage of Eq. (8) that for the complete description of steady-state creep rate dependence on temperature and applied stress only four parameters are enough. Obversely, the disadvantage of using parameters $T_{0}, \sigma_{0}, \dot{\varepsilon}_{0}$ and $E$ is low accuracy of their determination on the base of regression of experimental creep data using regression function (8) because the values of these parameters are far from the regions of testing temperatures, applied stresses and creep rates covering usual experimental conditions.

\section{Verification of new equation}

The derived equation (8) was verified using the test results of Sklenicka et al. $[5,6]$ obtained by studying creep of P91 steel. The experimental results and the fit using regression equation (8) are given in Fig. 1 (dependence on applied stress for various temperatures) and in Fig. 2 (dependence on temperature for various applied stresses, see converse reciprocal temperature axis on top of figure). In Fig. 2 only a part of results is plotted - only when a certain stress was applied at least at two temperatures.

Averages and standard deviations of regression parameters together with standard index of determination $r^{2}$ and corrected index of determination $r_{\text {corr }}^{2}$ are presented in Table 1 .

The points of intersection of straight lines are $(1478 \mathrm{MPa}$, $\left.10.8 \mathrm{~s}^{-1}\right)$ for Fig. 1 and $\left(1270 \mathrm{~K}, 10.8 \mathrm{~s}^{-1}\right)$ for Fig. 2. Parameter $E$ represents the apparent activation energy for the applied stress $\sigma=\sigma_{0} / \mathrm{e}=544 \mathrm{MPa}$. Because the values of regression parameters are substantially higher than typical values of strain rates, applied stresses, and temperatures, their determination cannot be very accurate in principle, see the values of standard deviations in Table 1 (but in spite of quite high values of standard deviations - relatively to the averages of regression parameters - the fit of experimental data is very successful which coincides with high values of the indexes of determination). The other consequence of this fact is that the values of regression parameters can hardly be interpreted 
as certain important quantities directly connected with the test material. Maybe only temperature $T_{0}$ (which is here $1270 \mathrm{~K} \approx$ $1000{ }^{\circ} \mathrm{C}$ ) could be interpreted as a very rough approximation of limit temperature at which the microstructure of test material changes by phase transformation.

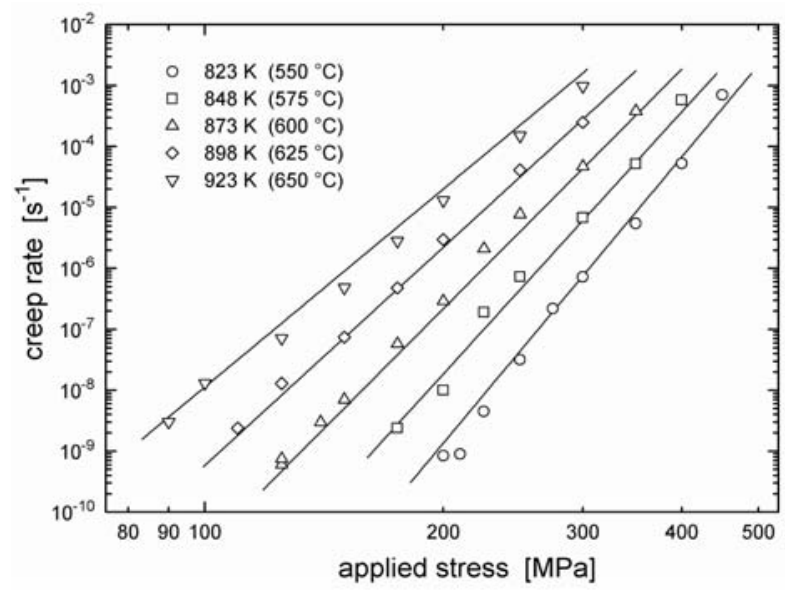

Fig. 1 Dependence of steady-state creep rate of P91 steel on applied stress for the given temperatures (Sklenicka et al. [5, 6]) and its regression using Eq. (8).

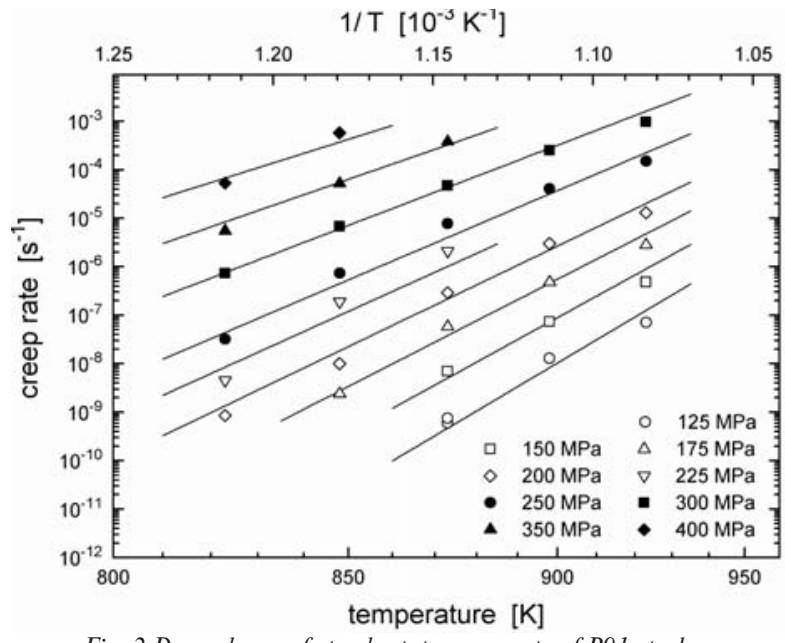

Fig. 2 Dependence of steady-state creep rate of P91 steel on temperature for the given applied stresses (Sklenicka et al. [5, 6]) and its regression using Eq. (8).

Regression parameters and indexes of determination

Table 1

\begin{tabular}{|c|c|c|c|c|c|}
\hline$\dot{\varepsilon}_{0}\left[\mathrm{~s}^{-1}\right]$ & $E[\mathrm{~kJ} / \mathrm{mol}]$ & $\sigma_{0}[\mathrm{MPa}]$ & $T_{0}[\mathrm{~K}]$ & $r^{2}[1]$ & $r_{\text {corr }}^{2}[1]$ \\
\hline $10.8 \pm 3.0$ & $303 \pm 36$ & $1478 \pm 329$ & $1270 \pm 66$ & 0.990026 & 0.989217 \\
\hline
\end{tabular}

Changing slopes in both figures mean that the stress sensitivity parameter is quite strongly dependent on temperature and that the apparent activation energy is quite strongly dependent on applied stress. The values of stress sensitivity parameter are not even approximately equal to an integer and they reach sometimes even 15 or more. It seems that the classical approach based on assumption of different particular mechanisms of creep deformation, represented by small integer constant values of the stress sensitivity parameter independent of temperature, should be modified and temperature dependence of this parameter should be considered, e.g. according to Eq. (7).

\section{Low temperature phenomena}

Eq. (10) can be also a starting point for the description of the dependence of yield stress on temperature and strain rate as well as for the description of stress relaxation curves, in both cases at temperatures lower than the usual temperatures of creep behaviour of studied material are. Only two following modifications are useful to do:

1. For both these phenomena often connected with substantially lower temperatures than creep it can be considered that $T_{0} \rightarrow \infty$ (i.e. $p=0$ ), which simplifies the description.

2. For lower temperatures the internal stress is necessary to introduce in the relation for applied stress which, on the other hand, a little complicates the description, i.e.

$$
\sigma=\sigma_{G}[\varepsilon, G(T)]+\sigma_{e f}(T, \dot{\varepsilon})
$$

where $\sigma_{G}$ is the internal stress connected with long-range obstacles and $\sigma_{e f}$ is the effective stress connected with short-range obstacles of dislocation motion. The internal stress is nearly temperatureindependent but it strongly depends on the development of dislocation structure, i.e. on the level of plastic deformation. On the other hand, short-range obstacles can be overcome with the assistance of thermal fluctuations and, therefore, the effective stress is strongly dependent on temperature and strain rate.

\subsection{Yield stress}

One of the most important stress values of test material is yield stress $\sigma_{y}$, i.e. the loading stress at which first substantial motion of dislocations appears and, in fact, plastic deformation starts to develop ( $\varepsilon \approx 0, \sigma_{G} \approx$ const.). Respecting both the low-temperature features mentioned above, the relation

$$
\sigma_{y}(T, \dot{\varepsilon})=\sigma_{G}+\sigma_{0} \exp \left(-\frac{R T}{E} \ln \frac{\dot{\varepsilon}_{0}}{\dot{\varepsilon}}\right) \equiv \sigma_{G}+\sigma_{0}\left(\frac{\dot{\varepsilon}}{\dot{\varepsilon}_{0}}\right)^{R T / L}
$$

for the description of yield stress dependence on temperature and strain rate can be written. Its validity can be verified by experimental results of Vlach et al. [7] studying the dependence of yield stress on temperature and strain rate for Cr-Mo steel used for pressure vessels. The yield stress was measured in the temperature range -196 to $+20{ }^{\circ} \mathrm{C}$, i.e. 77 to $293 \mathrm{~K}$, and the strain rate range 0.0014 to $11 \mathrm{~s}^{-1}$. Experimental points and fitting curves drawn in Fig. 3 show that the description of the dependence by Eq. (15) is very successful. 


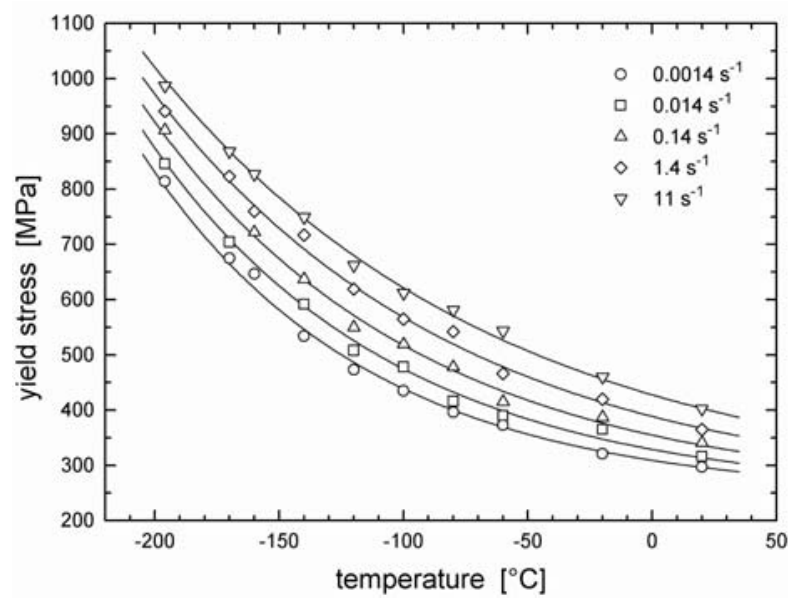

Fig. 3 Dependence of yield stress of Cr-Mo steel on temperature for the given strain rates (Vlach et al. [7]) and its regression using Eq. (15).

Another verification of Eq. (15) can be made using the experimental results of yield stress measurements of low-carbon steel published by Meyers [8]. The measurements were made in the temperature range 195 to $713 \mathrm{~K}$, i.e. -78 to $+440{ }^{\circ} \mathrm{C}$, and in the strain rate range $10^{-3}$ to $10^{5} \mathrm{~s}^{-1}$. For the verification, only the range 1 to $10^{3} \mathrm{~s}^{-1}$ was chosen, in which the measurements were made over the whole temperature range given above. Experimental results and their fit are drawn in Fig. 4. Also in this case the description of the dependence by Eq. (15) is very successful.

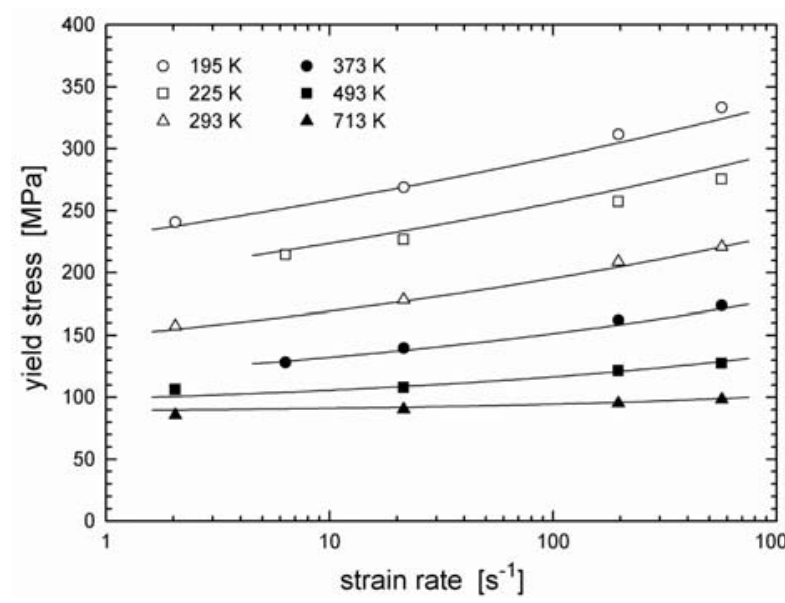

Fig. 4 Dependence of yield stress of low-carbon steel on strain rate for the given temperatures (Meyers [87) and its regression using Eq. (15).

Both Fig. 3 and Fig. 4 testify to the validity of Eq. (15), which was obtained on the basis of the newly derived Eq. (8).

\subsection{Stress relaxation}

The basis of the stress relaxation description consists in the proportionality between the strain rate and the rate of stress decrease

$$
\dot{\varepsilon}=-\frac{\dot{\sigma}}{M}
$$

where $M$ is modulus of elasticity of the system formed by a test bar and a testing device. Introducing this relation into Eq. (8), substituting $p=0$ and considering effective stress $\sigma$ ef instead of applied stress $\sigma$, a differential equation

$$
-\frac{d \sigma}{M}=\dot{\varepsilon}_{0}\left(\frac{\sigma-\sigma_{G}}{\sigma_{0}}\right)^{E / R T} d t
$$

is obtained, which can be solved by direct integration. Its solution is

$$
\sigma(t)=\sigma_{G}+\frac{\sigma(0)-\sigma_{G}}{(\alpha t+1)^{m}}
$$

where $\sigma(0)$ is the initial value of stress (for $t=0$ ) and parameters $\alpha$ and $m$ are temperature-dependent and they substitute the following more complicated expressions

$$
\begin{aligned}
& \alpha(T)=\frac{M \dot{\varepsilon}_{0}}{m(T) \sigma_{0}}\left[\frac{\sigma_{0}}{\sigma(0)-\sigma_{G}}\right]^{-1 / m(T)} \text { and } \\
& m(T)=\frac{R T}{E-R T}
\end{aligned}
$$

Eq. (18) is formally equivalent to the equation

$$
\sigma(t)=\sigma_{G}+K(t+a)^{-m}
$$

derived by Li [9] using considerations different from the considerations applied in this paper. Moreover, no further specifications of parameters $K$ and $a$ were given and no connection between parameter $m$ and temperature was described by $\mathrm{Li}$ [9].

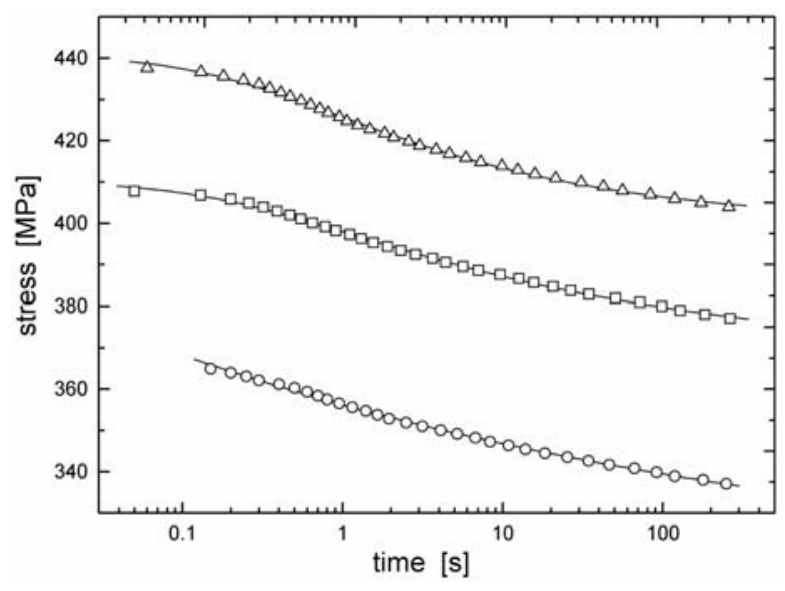

Fig. 5 Results of relaxation measurements of ferritic nodular cast iron (Kohout [10]) and its regression using Eq. (18). 
To verify Eq. (18), the relaxation curves of ferritic nodular cast iron determined by the author [10] were used, see Fig. 5. This figure (as well as all the previous figures) shows very good agreement between measured points and fitting curves.

The relaxation of many other metallic materials was studied with the same result: Eq. (18) provides for a better fit of experimental data than all the other equations used for this purpose.

\section{Discussion}

Eqs (8), (15), and (18) describe phenomena connected with creep, yield stress, and stress relaxation using common parameters $\dot{\varepsilon}_{0}, \sigma_{0}, E$ and $T_{0}$ or $\sigma_{G}$. In the case of creep temperature $T_{0}$ plays a significant role while internal stress $\sigma_{G}$ can be mostly neglected with respect to effective stress $\sigma_{e f}$. On the other hand, in yield stress effects and during stress relaxation nonzero internal stress $\sigma_{G}$ should be considered while the term $1 / T_{0}$ disappears considering $T_{0} \rightarrow \infty$. Naturally, parameters $\dot{\varepsilon}_{0}, \sigma_{0}$ and $E$ are common for all studied phenomena and their values can be determined by studying some of these phenomena. Then in the case of studying another phenomenon from those mentioned, the values of common parameters can be used e.g. for rough estimation or forecast of behaviour of studied material during the other phenomenon. An example is given by Kohout [10]: to determine the dependence of yield stress on temperature and strain rate it is sufficient to determine only strain rate dependence. The temperature dependence can be estimated on the basis of the value of parameter $E$ determined from exponent $m$ of the stress relaxation curves, see Eqs (15), (18) and (19). In the last resort only one test bar can be sufficient for a rough estimation of the temperature and strain rate dependence of yield stress, see [10].

\section{Conclusions}

1. Although using only four regression parameters, the newly derived relation describes the dependence of steady-state creep rate on temperature and applied stress better than the classically used Sherby relation does.

2. The newly derived relation respects a certain dependence of activation energy on applied stress and of the stress sensitivity parameter on temperature.

3. Based on the newly derived relation, the dependence of yield stress on temperature and strain rate can be deduced, which provides for a very good fit of experimental results.

4. Also the equation describing the relaxation curves better than the usually used equations do can be deduced from the newly derived relation.

5. Although being phenomenological, the presented consistent description of creep, yield stress, and relaxation issuing from the same base enables to study these phenomena commonly, using common set of parameters. The values of these parameters determined during studying one of these phenomena can be used in the description or prediction of the other phenomena.

\section{Acknowledgement}

The work presented in this paper has been supported by the Ministry of Defence of the Czech Republic (Research Plan No. MO0FVT0000404).

\section{References}

[1] CADEK, J.: Creep in Metallic Materials, Prague : Academia \& Amsterdam : Elsevier, 1988.

[2] GAROFALO, F.: Fundamentals of Creep and Creep Rupture in Metals, New York: Macmillan, 1965.

[3] SHERBY, O.D.: Acta Met., 1962, vol. 10, p. 135.

[4] KOHOUT, J.: New Description of Steady-state Creep Rate, Yield Stress, Stress Relaxation and their Interrelation, Materials Structure \& Micromechanics of Fracture IV (Materials Science Forum), 2005, vol. 482, pp. 319-322.

[5] SKLENICKA, V., KUCHAROVA, K., DLOUHY, A., KREJCI, J.: In: Materials for Advanced Power Engineering, Part I (ed. Coutsouradis, D.), Dordrecht : Kluwer Academic Publishers, 1994, p. 435.

[6] SKLENICKA, V., KUCHAROVA, K., KLOC, L.: In: Materials for Advanced Power Engineering 1998, Part I (eds Lecomte-Beckers, J. et al.), Julich : Forschungszentrum Julich, 1998, p. 233.

[7] VLACH, B., HOLZMANN, M., MAN, J.: Zvaranie [Welding], 2/1986, vol. 35, p. 35, ISSN 0044-5525 (in Czech).

[8] MEYERS, M.A.: Dynamic Behavior of Metals, New York : Wiley, 1994.

[9] LI, J.C.M.: Canadian Journal of Physics, 1967, Vol. 45, p. 493.

[10] KOHOUT, J.: Prediction of Temperature and Strain Rate Dependence of Yield Stress Using Test of One Bar at Room Temperature (in Czech), Materials Engineering, Vol. 4, 1997, pp. 1-9, ISSN 1335-0803. 